\title{
Pacific
}

Journal of

Mathematics

\section{THE BRAID INDEX OF GENERALIZED CABLES}

ROBERT Fones WiLliaMS AND ROBERT FONES WILLIAMS 


\title{
THE BRAID INDEX OF GENERALIZED CABLES
}

\author{
R. F. Williams
}

\begin{abstract}
If one knot is fashioned into another, by replacing each strand with $q$ strands, then something gets multiplied by $q$. What? The answer should not be overly dependent on how these strands are intertwined. We show that an invariant called the braid index is an answer. This proposition is apparently new. Another answer covered by our proof is the bridge number, though this was proved by Shubert in 1954. It was only with the advent of the Jones polynomial and its relatives in the mid 1980s, that much attention has been given to the braid index. For example, the knots obtained by repeated period doubling were shown to obey the multiplication rule, though no one seems to have thought of it this way. Their braid indices are powers of 2 . We first considered the current proposition in trying to show that a certain knot, known to have braid index 5 , could not be a two-cabling of anything.
\end{abstract}

Definitions. It is a classical result of Alexander [A] that any link, that is, a finite collection of smooth oriented simple closed curves embedded in Euclidian 3-space, can be isotoped into the (closure) of a braid on some number of strands, say $n$. By the braid index of a link we mean the least such number $n$.

The bridge number is the minimal number of local maxima for any smooth isotopic copy of a link $L$. See Shubert [S1; Satz 9, p. 283]. Our result follows as a corollary to Shubert's theorem in those cases for which these invariants are equal, since the bridge number is trivially seen to be less than or equal to the braid index.

We use $b(L)$ to denote the braid index (respectively bridge number) of an oriented link $L$. In the theorem below, we assume that each component of our link is knotted; this assumption is necessary in that (for example) any $(p, q)$ torus link is both a $p$-fold and a $q$-fold cabling of the unknot. For $p \neq q$ its braid index cannot be both $p$ and $q$. In fact its braid index is the lesser of $p$ and $q$ as can be seen, e.g., by the theorem of Morton [M] and Franks-Williams [FW]. That is, if $L$ is the closure of a positive braid on $p$ strands which has a full twist, then $L$ has braid index $p$. But, any $(p, q)$ torus link is a positive braid on $p$ strands as follows: denote the generators of the braid group $B_{p}$ by $2,3, \ldots, p$. The $(p, q)$ torus link $L_{p q}$ is the closure of the braid $\beta=(2,3, \ldots, p)^{q}$. Since the full twist 

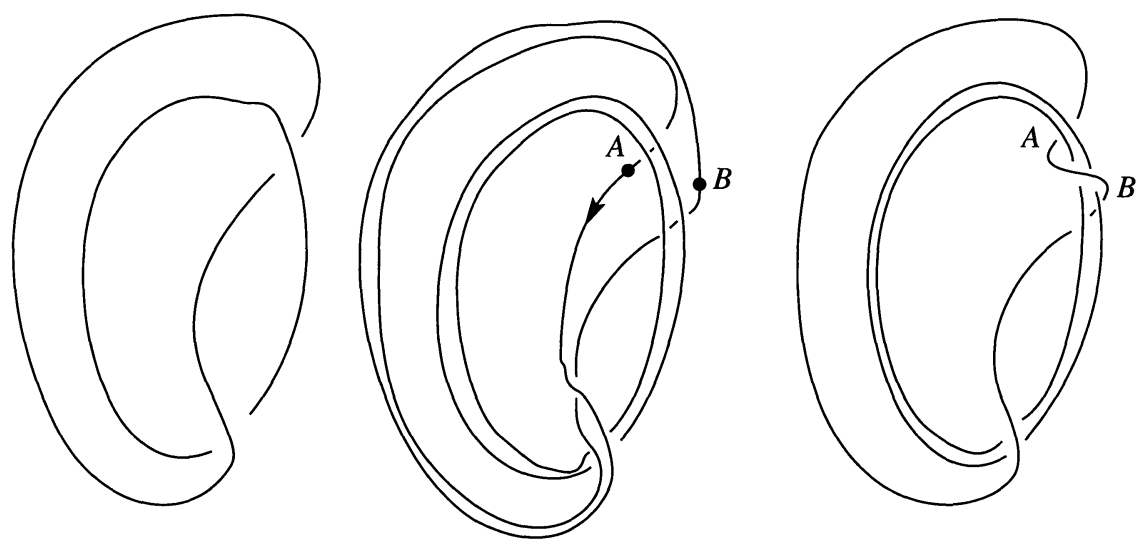

FIGURE 1. A 2-cabling of the Hopf link

is $(2,3, \ldots, p)^{p}, \beta$ contains a full twist, in case $p \leq q$. For as the closure of a braid in $B_{p}$ the $(p, q)$ torus link is positive, and if $p \leq q$ it contains a full twist. Another example: the Hopf link (i.e., the link consisting of two simply linked circles) has a 2-cabling of braid index 3. For example, cable one component by 2 parallel circles, the other by the unknot, just as one "doubles" a rubber band. The resulting 2-cabling has braid index $\leq 3$, as one sees by relaxing the "rubber band." Having 3 components, its braid index is $\geq 3$, and thus 3 .

Start with the Hopf link, the first diagram in Figure 1. Next, 2-cable it getting the second diagram. Then the long arc from $A$ to $B$ in the second diagram is moved to the short diagonal stretch in the final picture. In terms of the braid generators $1,2,3$ of $B_{4}, 213212312 \rightarrow$ $213121312 \rightarrow 211323112 \rightarrow 211232112 \rightarrow 211221112$.

Below we work with generalized cablings of knots in $\mathbf{R}^{3}$. By a generalized $q$-cabling of a link $L$ we mean a link $L^{\prime}$ contained in the interior of a tubular neighborhood $L \times D^{2}$ of $L$ such that

(a) each fiber $D^{2}$ intersects $L^{\prime}$ transversely in $q$ points; and

(b) all strands of $L^{\prime}$ are oriented in the same direction as $L$ itself.

Note that any link which is arranged as a braid on $p$ strands, is a generalized $p$ cabling of the unknot, since it can be put inside the unknotted torus, always travelling in the same direction. Thus the figure eight knot is a generalized 3-cabling of the unknot, which cannot be a cabling of the unknot, as it is easily seen to not be a torus knot.

We shorten this phrase to g-cabling or $q$-g-cabling below. The term cabling is reserved for the g-cablings which are actually on the boundary torus itself. Part of the interest in our proof is its simplicity and the fact that it works for both invariants with no change at all. It is a 
pleasure to thank my colleagues, John Berge, Cameron Gordon, Ray Lickorish, and John Luecke for helpful conversations. We would like to thank the referee for bringing to our attention a related result of Schubert [S2, Satz 1, p. 275], reviewed by R. Fox, in Math. Reviews 17, p. 298.

THEOREM 1. The braid index (respectively bridge number) is multiplicative under generalized cabling. In detail, if $L$ is a link with each component a non-trivial knot and $L^{\prime}$ is a generalized q-cabling of $L$ then

$$
b\left(L^{\prime}\right)=q b(L)
$$

Proof. Clearly

$$
b\left(L^{\prime}\right) \leq q b(L),
$$

since the obvious cabling of $L$ is a braid of $q b(L)$ strands. (Respectively, is a plat with bridge number $q b(L)$.) We proceed to prove the other inequality, and to simplify notation, prove it first in the case that our link $L$ has only one component and thus is a knot, $K$, with generalized $q$-cabling $K^{\prime}$. Assume on the contrary that

$$
b\left(K^{\prime}\right)=b<q b(K) .
$$

We work in the smooth setting, and assume $K^{\prime}$ is presented as a $q$-g-cabling of $K$ in a small tubular neighborhood $N$ of $K$. This neighborhood is a solid torus neighborhood $N$ of the knot $K$. Under our assumption, we know there is a global diffeomorphism $\Phi: \mathbf{R}^{3} \rightarrow$ $\mathbf{R}^{3}$ of compact support such that

(a) $\Phi\left(K^{\prime}\right)$ has exactly $b$ maxima and $b$ minima which are nondegenerate and no other singularities;

(b) the "height" function $h$ : bdry $\Phi(N) \rightarrow \mathbf{R}$ is a Morse function, i.e., $h$ has finitely many singularities, all non-degenerate.

We are given condition (a). But since this condition is generic, by a small perturbation, we may assume (b).

Let $T^{2}=$ bdry $\Phi(N)$. By (b) the intersection of horizontal planes with our torus $T^{2}$ consists mostly of disjoint simple closed curves. The finitely many exceptional intersections are at singularities of $h$ and have in addition, an isolated point or "figure eight." These latter consist of two simple closed curves, which intersect in a single point.

A simple closed curve in $T^{2}$ which bounds a disk in $T^{2}$ will be called trivial. For a non-trivial $J \subset T^{2}$, we follow tradition and say $J$ is a meridian, provided $J$ bounds a disk in $\Phi(N)$. Note that for 
a meridian $J$ the linking number $l(K, J)=1$ and that for our $q$ g-cabling $K^{\prime}, l\left(K^{\prime}, J\right)=q$, these numbers being determined up to sign.

REMARK 1. If $J$ is a simple closed curve lying in $T^{2} \cap \pi$, where $\pi$ a horizontal plane, then $J$ is either a meridian of $T^{2}$ or is trivial in $T^{2}$.

Proof. Since $J$ bounds a disk in the plane, it bounds a disk in $\mathbf{R}^{3}$. But the only such simple closed curves on $T^{2}$ are meridians or those that bound disks in $T^{2}$, because $K$ is knotted.

REMARK 2. There is a horizontal plane intersecting $T^{2}$ in a meridian.

Proof. This is Morse theory at its simplest: let $T(x)$ consist of all those points $p \in T^{2}$ of height $h(p) \geq x$, for $x \in \mathbf{R}$. Then $T(x)$ is empty, for $x$ large. As we decrease $x, T(x)$ first becomes a point, then a disk, changing its topological type only at singularities of $h$. Thus under the assumption that we never meet a meridian, only three things can happen: a new disk is added, two disks are joined making one less disk, or (a priori, only) an old disk is capped off with a new disk, forming a sphere. Thus, for small $x$ we get $T^{2}=T(x)$ is the union of finitely many disjoint disks and disjoint spheres, which is absurd.

Let $J$ be a meridian of $T^{2}$ lying on a horizontal plane $\pi$. We can and do assume $T^{2}$ is transverse to $\pi$ and that $J$ is innermost among meridia. Then $J$ bounds a disk $d \subset \pi$ and by our remarks above, $d$ separates $K^{\prime}$ into a number of components $\left\{C_{i}\right\}$. Some $C_{i}$ 's are non-trivial, in that there is an $\operatorname{arc} D_{i}$ in the interior of $J$ such that

$$
C_{i} \cup D_{i} \cup\{\text { end points }\}=\widehat{K}_{i}
$$

traverses $\Phi(N)$, the interior of $T^{2}$, longitudinally. Others may be trivial in that such a $\widehat{K}_{i}$ bounds a disk in $\Phi(N)$. However

REMARK 3. There are (at least) $q$ non-trivial $\widehat{K}_{i}$.

Proof. The signed linking number of $L$ with $J$ is $q$. The trivial $\widehat{K}_{i}$ add nothing as they bound in $\Phi(N)$ and each non-trivial $\widehat{K}_{i}$ counts +1 . 


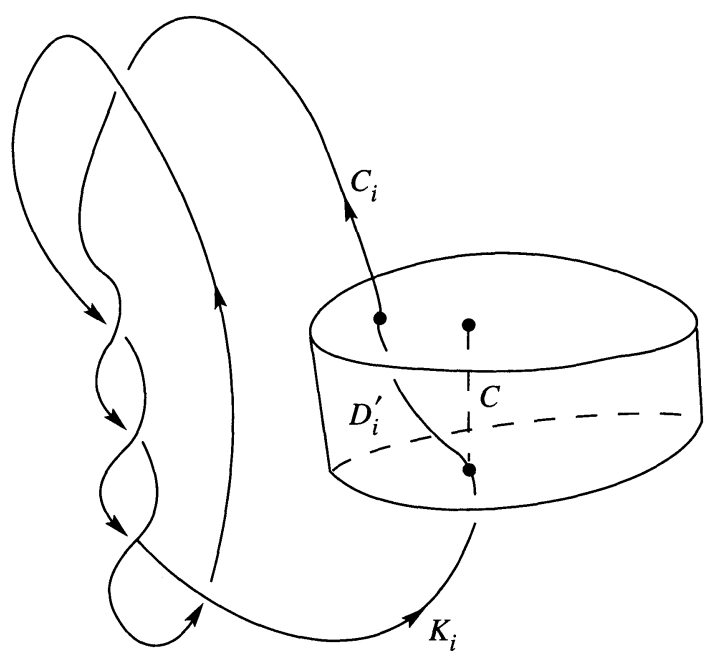

FIGURE 2

Discard the trivial $\widehat{K}_{i}$. The remaining $\widehat{K}_{i}$ are not presented as braids (respectively plats) only because the $D_{i}$ are horizontal. However, this is easy to remedy. Choose another plane $\pi^{\prime}$ just below $\pi$ so that together they cut an annulus $A$ off of $T^{2}$ whose other boundary curve $J^{\prime}$ is essentially parallel to $J$. Then form

$$
\left(C_{i}-C\right) \cup D_{i}^{\prime} \cup\{\text { end points }\}=K_{i},
$$

where $D_{i}^{\prime}$ is a rising arc lying between the planes replacing a small arc $C \subset C_{i}$, which is discarded. It is clear that the $D_{i}^{\prime}$ can be taken to be disjoint, so that the $K_{i}$ are disjoint as well. See Figure 2.

Thus we have determined $q$ knots $K_{1}, K_{2}, \ldots, K_{q}$, each isotopic to $K$. Now compute

$$
\begin{aligned}
\sum_{i=1}^{q}\left\{\text { number of max of } K_{i}\right\} & \leq\left\{\text { number of } \max \text { of } K^{\prime}\right\} \\
& =b\left(K^{\prime}\right)=b<q b(K) .
\end{aligned}
$$

But the $K_{i}$ are mutually disjoint. It follows that one of the $K_{i}$ has fewer than $b(K)$ maxima and thus is a braid on fewer than $b(K)$ strands (respectively, plat with fewer than $b(K)$ bridges). This contradicts the fact that each $K_{i}$ is isotopic to $K$ and hence has the same braid index (respectively bridge number) as $K$. This completes the proof, in case of a simple knot, $K$.

We turn to the general case in which our link consists of several knots. We introduce notation as follows: $L$ is a link whose components are knots (each is knotted) $K_{1}, K_{2}, \ldots, K_{k}$, and $L^{\prime}$ is a 
$q$-g-cabling of $L$. We then assume for contradiction that

$$
b\left(L^{\prime}\right)=b<q b(L) .
$$

Then $L^{\prime}=K_{1}^{\prime} \cup K_{2}^{\prime} \cup \cdots \cup K_{k}^{\prime}$ where for each $j, K_{j}^{\prime}$ is a $q$-g-cabling of $K_{j}$ lying in a small tubular neighborhood $N_{j}$ of $K_{j}$. Under our assumption, there is a global diffeomorphism $\Phi: \mathbf{R}^{3} \rightarrow \mathbf{R}^{3}$, with compact support, so that $\Phi\left(L^{\prime}\right)$ is represented by a braid (respectively, a plat) with $b$ maxima.

As before, we may adjust the neighborhoods $\Phi\left(N_{j}\right)$ slightly, so that the torus, $\partial \Phi\left(N_{j}\right)=T_{j}$ has a Morse height function, for $j=$ $1,2, \ldots, k$. We must deal with all of the $T_{j}$ simultaneously, since neither the braid index nor the bridge number is additive over components. Fix $j$. Then just as before there is a plane $\pi_{j}$ so that $T_{j} \cap \pi_{j}$ is a meridian of $T_{j}$. Proceeding as before, construct $q$ knots, $K_{j 1}, K_{j 2}, \ldots, K_{j q} \subset \Phi\left(K_{j}\right)$, which are disjoint, and except for short rising arcs, are subsets of $\Phi\left(K_{j}\right)$. Having done this for each $j=1, \ldots, k$, define

$$
L_{i}=K_{1 i} \cup K_{2 i} \cup \cdots \cup K_{k i}, \quad i=1, \ldots, q .
$$

Now compute

$$
\begin{aligned}
\sum_{i=1}^{q}\left\{\text { number of max of } L_{i}\right\} & \leq\left\{\text { number of } \max \text { of } L^{\prime}\right\} \\
& =b\left(L^{\prime}\right)=b<q b(L) .
\end{aligned}
$$

But the $L_{i}$ are mutually disjoint. It follows that one of the $L_{i}$ has fewer than $b(L)$ maxima and thus is a braid on fewer than $b(L)$ strands (respectively, plat with fewer than $b(L)$ bridges). This contradicts the fact that each $L_{i}$ is isotopic to $L$ and hence has the same braid index (respectively bridge number) as $L$. This completes the proof, in case of a simple knot, $L$.

\section{REFERENCES}

[A] J. W. Alexander, A lemma on systems of knotted curves, Proc. Nat. Acad. Sci. U.S.A., 9 (1923), 93-95.

[F-W] J. Franks and R. Williams, Braids and the Jones polynomial, Trans. Amer. Math. Soc., 303, no. 1, (1987), 97-108.

[M] H. Morton, Closed braid representatives for a link and its 2-variable polynomial, preprint, Liverpool University, 1985. 
[S1] H. Shubert, Über einer numerische Knoteninvariante, Math. Zeit. Bd., 61 (1954), 245-288.

[S2] _ Knotten und Vollringe, Acta Math., 90 (1953), 131-276.

Received April 15, 1990 and in revised form November 1, 1991. Supported in part by a grant from the National Science Foundation. We wish to thank the Aspen Center for Physics for its hospitality.

The University of TeXas at Austin AusTin, TX 78712 



\section{PACIFIC JOURNAL OF MATHEMATICS EDITORS}

\author{
V. S. VARADARAJAN \\ (Managing Editor) \\ University of California \\ Los Angeles, CA 90024-1555 \\ vsv@math.ucla.edu \\ Herbert Clemens \\ University of Utah \\ Salt Lake City, UT 84112 \\ clemens@math.utah.edu \\ F. Michael Christ \\ University of California \\ Los Angeles, CA 90024-1555 \\ christ@math.ucla.edu \\ THOMAs ENRIGHT \\ University of California, San Diego \\ La Jolla, CA 92093 \\ tenright@ucsd.edu
}

\author{
Nicholas ERCOLANI \\ University of Arizona \\ Tucson, AZ 85721 \\ ercolani@math.arizona.edu \\ R. FINN \\ Stanford University \\ Stanford, CA 94305 \\ finn@gauss.stanford.edu \\ VAUGHAN F. R. Jones \\ University of California \\ Berkeley, CA 94720 \\ vfr@math.berkeley.edu \\ Steven KerckhofF \\ Stanford University \\ Stanford, CA 94305 \\ spk@gauss.stanford.edu
}

\author{
C. C. MOORE \\ University of California \\ Berkeley, CA 94720
}

Martin ScharlemanN

University of California

Santa Barbara, CA 93106

mgscharl@henri.ucsb.edu

Harold Stark

University of California, San Diego La Jolla, CA 92093

\section{R. ARENS \\ ASSOCIATE EDITORS}

\begin{tabular}{lccc} 
R. ARENS & $\begin{array}{c}\text { E. F. BECKENBACH } \\
(1906-1982)\end{array}$ & B. H. NEUMANN & \multicolumn{1}{c}{ F. WOLF } \\
$(1904-1989)$ & K. YoshIDA \\
SUPPORTING & INSTITUTIONS \\
UNIVERSITY OF ARIZONA & UNIVERSITY OF OREGON \\
UNIVERSITY OF BRITISH COLUMBIA & UNIVERSITY OF SOUTHERN CALIFORNIA \\
CALIFORNIA INSTITUTE OF TECHNOLOGY & STANFORD UNIVERSITY \\
UNIVERSITY OF CALIFORNIA & UNIVERSITY OF HAWAII \\
MONTANA STATE UNIVERSITY & UNIVERSITY OF TOKYO \\
UNIVERSITY OF NEVADA, RENO & UNIVERSITY OF UTAH \\
NEW MEXICO STATE UNIVERSITY & WASHINGTON STATE UNIVERSITY \\
OREGON STATE UNIVERSITY & UNIVERSITY OF WASHINGTON \\
\hline
\end{tabular}

The Supporting Institutions listed above contribute to the cost of publication of this Journal, but they are not owners or publishers and have no responsibility for its content or policies.

Mathematical papers intended for publication in the Pacific Journal of Mathematics should be in typed form or offset-reproduced (not dittoed), double spaced with large margins. Please do not use built up fractions in the text of the manuscript. However, you may use them in the displayed equations. Underline Greek letters in red, German in green, and script in blue. The first paragraph must be capable of being used separately as a synopsis of the entire paper. In particular it should contain no bibliographic references. Please propose a heading for the odd numbered pages of less than 35 characters. Manuscripts, in triplicate, may be sent to any one of the editors. Please classify according to the 1991 Mathematics Subject Classification scheme which can be found in the December index volumes of Mathematical Reviews. Supply name and address of author to whom proofs should be sent. All other communications should be addressed to the managing editor, or Elaine Barth, University of California, Los Angeles, California 90024-1555.

There are page-charges associated with articles appearing in the Pacific Journal of Mathematics. These charges are expected to be paid by the author's University, Government Agency or Company. If the author or authors do not have access to such Institutional support these charges are waived. Single authors will receive 50 free reprints; joint authors will receive a total of 100 free reprints. Additional copies may be obtained at cost in multiples of 50 .

The Pacific Journal of Mathematics (ISSN 0030-8730) is published monthly except for July and August. Regular subscription rate: $\$ 190.00$ a year (10 issues). Special rate: $\$ 95.00$ a year to individual members of supporting institutions.

Subscriptions, orders for numbers issued in the last three calendar years, and changes of address should be sent to Pacific Journal of Mathematics, P.O. Box 969, Carmel Valley, CA 93924, U.S.A. Old back numbers obtainable from Kraus Periodicals Co., Route 100, Millwood, NY 10546.

The Pacific Journal of Mathematics at P.O. Box 969, Carmel Valley, CA 93924 (ISSN 0030-8730) is published monthly except for July and August. Second-class postage paid at Carmel Valley, California 93924, and additional mailing offices. Postmaster: send address changes to Pacific Journal of Mathematics, P.O. Box 969, Carmel Valley, CA 93924.

PUBLISHED BY PACIFIC JOURNAL OF MATHEMATICS, A NON-PROFIT CORPORATION Copyright (C) 1992 by Pacific Journal of Mathematics 


\section{PACIFIC JOURNAL OF MATHEMATICS}

Volume $155 \quad$ No. $2 \quad$ October 1992

Invariant subspaces and harmonic conjugation on compact abelian

201 groups

NAKHLE HABIB ASMAR, EARL ROBERT BERKSON and

ThOMAS Alastair GiLlESPIE

Cobcat and singular bordism

A. K. DAS and S. S. KHARE

Poles of Eisenstein series on $\mathrm{SL}_{n}$ induced from maximal parabolics

PAUL FEIT

On the postulation of 0-dimensional subschemes on a smooth quadric

251

SAlvatore GiUfFrida, Renato MagGioni and AlFio

RAGUSA

The Euler class for "piecewise" groups

Peter Abraham GREenberg

$L^{p}$-Fourier transforms on nilpotent Lie groups and solvable Lie groups 295 acting on Siegel domains

JUNKO INOUE

Fuchsian moduli on a Riemann surface-its Poisson structure and

Poincaré-Lefschetz duality

KATSUNORI IWASAKI

Interpolation between $H^{p}$ spaces and noncommutative generalizations. 341 I

\section{GILLES PISIER}

The braid index of generalized cables

ROBERT FONES WILLIAMS and ROBERT FONES WILLIAMS $B M O$ and Hankel operators on Bergman spaces 